\title{
Food allergy unplugged
}

The prevalence of food allergy is on the increase. Yet how susceptible individuals become sensitized to food antigens remains poorly understood. Now, a report in The Journal of Experimental Medicine shows an important role for interleukin-9 (IL-9) and mastcell-mediated gut leakiness in the induction of oral antigen hypersensitivity in mice.

IL-9 has previously been implicated in T helper $2\left(\mathrm{~T}_{\mathrm{H}} 2\right)$-cell driven allergic reactions in the airways, in which it has an important role in IgE production and mast-cell activation. So, Forbes et al. looked to see whether IL-9 might be similarly involved in allergic reactions in the gut induced by sensitization with ovalbumin (OVA) and subsequent oral challenge with OVA. Indeed, Il9 mRNA levels in the small intestine were found to increase in wild-type mice with oral-antigeninduced intestinal anaphylaxis. Moreover, the intestinal anaphylaxis, characterized by mastocytosis, mast-cell activation and diarrhoea, was attenuated in IL-9-deficient mice. This attenuated phenotype was associated with fewer intestinal mast cells and lower levels of mast-cell protease 1 but not with a diminished $\mathrm{T}_{\mathrm{H}}$ 2-type response.

The role of IL-9 in increasing intestinal mast-cell numbers was confirmed using mice that overexpressed IL- 9 specifically in the enterocytes of the small intestine. A comparison of the genes expressed

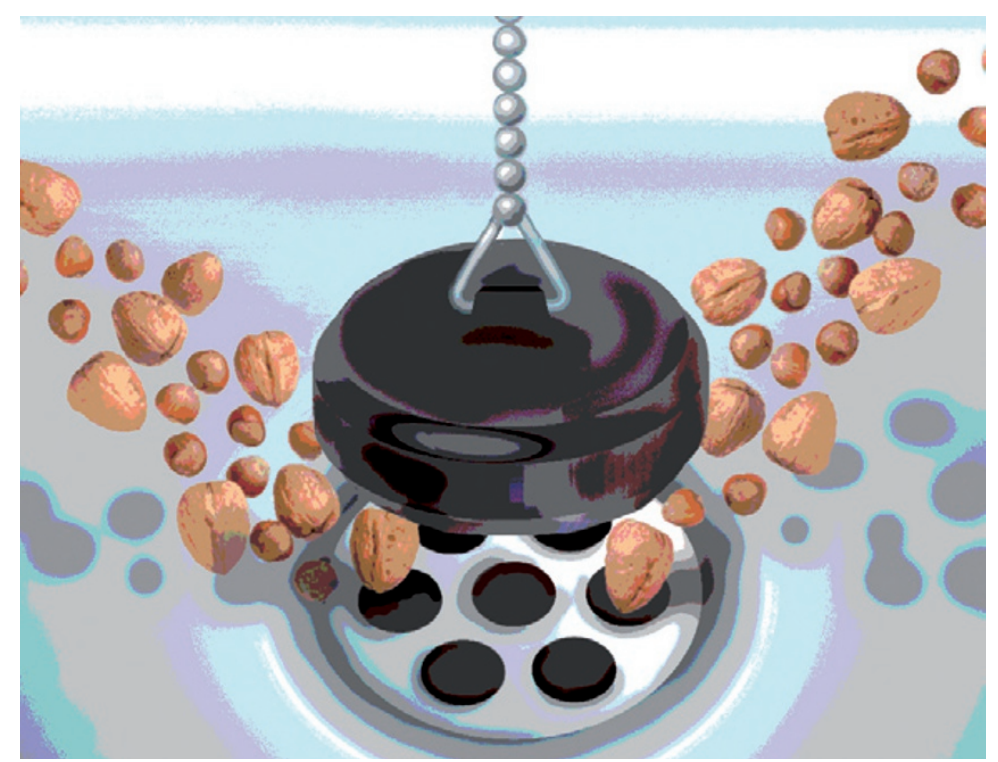

in the intestine of these IL-9-overexpressing mice and of wild-type mice with oral-antigen-induced intestinal anaphylaxis revealed a similar profile, suggesting that IL-9 overexpression in the intestine is sufficient to promote an intestinal anaphylaxis-like phenotype.

Interestingly, a prominent feature in both groups of mice was increased permeability of the intestine ('leaky gut'), a factor thought to predispose to gastrointestinal disease in humans. Consistent with this being a susceptibility factor, mice overexpressing IL-9 in the gut were more susceptible to oral-antigen-induced intestinal anaphylaxis, such that prior sensitization with OVA was not necessary for the induction of allergic disease following oral OVA challenge. Finally, a link between the leaky gut phenotype and mast cells was confirmed, by showing that mast-cell depletion abrogated the increased intestinal permeability in IL-9-overexpressing mice.

So, together these observations support a mechanism whereby IL-9 overexpression predisposes mice to oral antigen hypersensitivity by acting as a potent inducer of mastocytosis, which in turn increases intestinal permeability.

Lucy Bird

ORIGINAL RESEARCH PAPER Forbes, E. E. et al. IL-9- and mast cell-mediated intestinal permeability predisposes to oral antigen hypersensitivity. J. Exp. Med. 31 March 2008 (doi:10.1084/jem.20071046) 